Southern Illinois University Carbondale

OpenSIUC

Fall 10-1-2016

\title{
A comparison of three emerging online government 3D printing resources: NASA 3D Resources, Smithsonian X3D, and the NIH 3D Print Exchange
}

Jennifer J. Horton

Southern Illinois University Carbondale, jhorton@lib.siu.edu

Jian Anna Xiong

Southern Illinois University Carbondale, axiong@lib.siu.edu

Follow this and additional works at: http://opensiuc.lib.siu.edu/morris_articles

\section{Recommended Citation}

Horton, Jennifer J. and Xiong, Jian Anna. "A comparison of three emerging online government 3D printing resources: NASA 3D Resources, Smithsonian X3D, and the NIH 3D Print Exchange." The Charleston Advisor 18, No. 2 (Fall 2016): 5-10(6). doi:10.5260/ chara.18.2.5. 


\section{\$5 A Comparison of Three Emerging Online Government 3D Printing Resources NASA 3D Resources, Smithsonian X3D,
and the NIH 3D Print Exchange}

doi:10.5260/chara.18.2.5

Date of Review: July 1, 2016

NASA 3D Composite Score:
Smithsonian X3D Composite Score:
NIH 3D Print Exchange Composite Score: $\quad \star \star \star \star \star \star ~$

$<$ jhorton@lib.siu.edu> <axiong@lib.siu.edu>

\section{Abstract}

United States governmental agencies, departments, and affiliates, are increasingly making 3D printable models available to the general public online. On March 20, 2014, a memorandum was sent out to United States executive departments and agencies about improving access to the government's scientific collections (Holdren, 2014). This memorandum, from the White House Office of Science and Technology Policy (OSTP), not only addressed physical collections, but specifically mentioned digital 3D models. On the White House's blog, several reasons were given for making these collections more accessible including the idea that these collections are "... treasure troves of information ripe for exploration and learning" (Stebbins \& Lieberman, 2014).

Several areas of the United States government have begun making $3 \mathrm{D}$ models available to the general public or were already doing so at the time the memorandum was released. Three of the largest collections are from the National Aeronautics and Space Administration (NASA), the Smithsonian Institution, and the National Institutes of Health (NIH). Two of these, the NIH and NASA, are government agencies while the Smithsonian Institution is a Trust Instrumentality of the United States (Legal Nature, 2016). These sites offer down- loadable, printable files in addition to educational resources. The Smithsonian's website even states it is "the end of 'do not touch.", (Getting Started, 2016).

This review focuses on the $3 \mathrm{D}$ printing sections of these three websites including the models, supplementary materials, and resources.

\section{Pricing Options}

All three resources are free websites and offer free content including downloadable 3D models suitable for printing.

\section{Product Overview/Description \\ NASA 3D Resources}

NASA has developed a 3D resources website which makes available several collections of 3D data and materials including 3D models, 3D models optimized specifically for 3D printing, 3D textures and visualizations, and images. The website can be found at $<\mathrm{https}$ ://nasa3d.arc.nasa.gov $>$. Maintained by NASA, the site has many agency contributors including the Jet Propulsion Laboratory, Ames Research

\section{At a Glance Comparative Review Scores}

The maximum number of stars in each category is 5 .

\begin{tabular}{lccc} 
& NASA 3D & Smithsonian X3D & NIH 3D Print Exchange \\
\hline Composite: & $\star \star \star 1 / 4$ & $\star \star \star 3 / 4$ & $\star \star \star \star$ \\
Content: & $\star \star \star 1 / 2$ & $\star \star \star \star$ & $\star \star \star \star$ \\
User Interface/Searchability: & $\star \star \star$ & $\star \star \star 1 / 2$ & $\star \star \star \star$ \\
Pricing: & N/A & N/A & N/A \\
Purchase/Contract Options: & N/A & N/A & N/A \\
\hline
\end{tabular}


Center, Johnson Space Center, and Goddard Space Flight Center. In addition, Ball Aerospace and the California Institute of Technology are primary authors of some models.

The models found through NASA are free and without copyright. There are special guidelines, though, when using the NASA insignia found on some models. Models on the site include such varied subject matter as topographic Apollo landing sites; models of satellites, telescopes, and rovers; models of hurricanes; models of asteroids; and NASA insignia and medallions. Also included are 3D models that were actually printed by astronauts aboard the International Space Station, including a jar and a wrench. The site is geared to the public. Much of the website is focused on education and includes news articles, worksheets, and other educational resources relating to the various objects and images.

The files that are 3D printable include a 3D Model Viewer rendered by GitHub where users can view and manipulate the file. For some of the $3 \mathrm{D}$ visualizations, there are specific system and browser requirements. These requirements can be found under the individual visualization's description. All of the 3D models available for printing are made available in STL file format. Some can also be found as OBJ models or in a format to use with the open source software Blender.

\section{Smithsonian X3D}

The first of these three 3D model repositories, Smithsonian X3D, began in November 2013 (Printing Up, 2014). The website can be found at $<\mathrm{http}: / / 3 \mathrm{~d}$.si.edu/ $>$. The site includes several downloadable models of artifacts from the many Smithsonian museums and collections. The organization's Digitization Program Office oversees the website, establishes workflows, scans specific artifacts, and makes those models available to the general public. The site was developed with Autodesk and is sponsored by many technology companies including Autodesk and 3DSystems. Items scanned and presented in this collection include such things as Abraham Lincoln life masks, a Woolly Mammoth skeleton, Amelia Earhart's Flight Suit, and a funerary relief bust of Haliphat.

The site not only includes the printable 3D model of the artifacts, but for many, it includes supplementary materials including historical articles and related images from the Smithsonian's collection. There are also special sections devoted specifically to education, particularly K-12 education.

\section{NIH 3D Print Exchange (3DPX)}

The NIH 3D Print Exchange is a website which is a collaborative effort between the National Institute of Allergy and Infectious Diseases, the Eunice Kennedy Shriver National Institute for Child Health and Human Development, and the National Library of Medicine. The site, found at $<\mathrm{http} / / / 3 \mathrm{~d}$ print.nih.gov/ $>$, provides biomedical 3D printable models focusing on cells, bacteria, viruses, molecules, and anatomical structures. In addition to providing NIH created 3D models, this website allows users to submit their own biomedical 3D models by simply sharing the model or creating the model from raw data returned from the Protein Data Bank or the Electron Microscopy Data Bank.

This site has a wide range of suggested users. The website specifically mentions researchers, educators and students, presenters, and 3D printing enthusiasts. Numerous educational materials, tutorials, and opportunities for users to create and share 3D models are included. There are several NIH created videos and teaching supplements accessible on the site.

\section{User Interface/Navigation/Searching NASA 3D Resources}

NASA's 3D site has a simple and straightforward interface. Even the colors of the site are minimal and based around blues and greys. There are sections for the different types of available resources. Not everything on the site is optimized for 3D printing, but the site makes it simple to differentiate between the models suitable for 3D printing and those which are not. The main sections of the website are highlighted on the homepage with related links also visible, but not distracting. It is easy to determine the number of models and view both the latest models added to the site and any featured models as well.

The site also works well on mobile devices. Items can be searched easily on a mobile device and the models can be viewed with the $3 \mathrm{D}$ model viewer as well.

The site consists of a simple search box and a browsable A-Z list of $3 \mathrm{D}$ printable items. The $\mathrm{A}-\mathrm{Z}$ list can be sorted by name or by the date the model was added to the site, making it easy to determine if any new models have been made available. There is no advanced search, but as of the date of this review, there are not enough models available that this is a needed feature. It is easy to browse through the models as each has a title and thumbnail photo available in the browsable list and in the search results list. By clicking on one of the models, the user will find linked key words that can be used to produce search results lists of other models tagged with those key words. Examples of some of these key words include Mars, Landing Site, or Satellite.

Each 3D printable model on the site has useful information about the actual NASA object the model is based upon and the 3D model itself. For each object, the relevant related mission is given. There is also a link providing more information about that particular mission. For instance, a model of the Apollo 11 Landing Site directs users to NASA's Apollo Missions website which contains information on the Apollo program, individual missions, and the astronauts involved. As for the 3D model, there is a 3D Model Viewer where the user can see the model on a platform, manipulate the model, view it in either wireframe view, surface angle view, or as a solid. This is useful because not all 3D model sites provide users a way to see the model before they actually download it. Downloading the printable version of the 3D model is very simple. There are buttons with links to downloading the appropriate file. Some models have only STL files, while others have other 3D formats such as $\mathrm{OBJ}$ or Blender files.

In addition to the $3 \mathrm{D}$ models, the site contains many related links pertaining to the related missions, 3D printing generally, or 3D printing by NASA researchers and astronauts. There are also notices on the site asking for users to share with NASA their experiences printing the models.

\section{Smithsonian X3D}

The Smithsonian's 3D site is attractive and eye-catching. Featured models are displayed in color along with a video showcasing the model from all angles. There are also several parts of the website highlighted on the homepage with thumbnail views. These include 
videos, a section for educators, and a getting started section. The project is very active on social media and a display of the group's Twitter feed is prominently displayed.

There are also several tabs with links to other sections of the site including the models, tours of the models, a video gallery, and a link to information about a 2013 Conference sponsored by the Smithsonian $\mathrm{X} 3 \mathrm{D}$ project. There are also repeat links to the educators section and the about section of the site included in these tabs.

In order to download any of the models, a free account must be established. Users can register with the link in the upper right hand corner of the screen or when prompted by the Download button for each individual model. Once registered, users will need to log in and agree to the terms of use in order to download specific 3D model of the objects.

The site appears to be geared to education and highlighting American history. Much more is provided than just the actual 3D models. Some models contain what are referred to as Tours. These tours focus on different topics depending on the artifact. Some look at the history of the artifact, the artist, the artifact's historical time period, the conservation process, or the scanning and digitization process. Each model has a link to the Tours, but it is not always intuitive if a tour is actually available or not. One has to click on the object to determine if a tour is available related to that model. If not, the page is simply empty.

There is no search capability to find models on this site. Users are only able to browse the models. There is not a large number of models at this point, so this does not seem to hinder the user's ability to find an appropriate model. By clicking on the Browse Models tab, users will see titles and images of the models. They are also given the specific Smithsonian organization that provided the object such as the National Museum of Natural History or the National Air and Space Museum. Also for each model, users can view available tours, download the model, and see the model in an online model viewer. In order to use the model viewer, users need to click on the button Info or click on the image of the object. This is not straightforward and some users may miss this feature.

In order to run the 3D web applications on the site, the user must have a browser that supports WebGL. WebGL is a JavaScript API that allows for 3D graphics, including models, to be viewed in web browsers. (Danchilla, 2012). Most up to date browsers will be supported in Windows and Apple OS, but WebGL will likely need to be enabled if using Safari. Apple iOS devices like iPhones and iPads do not permit the use of WebGL, but it is fully supported on Google Android devices (Prerequisites, 2016).

Each model can be viewed on the site's 3D Exploration Tool, which is essentially a 3D model viewer. The site does state at this time that it is a Beta tool and they request feedback and bug reports. This model viewer, powered by Autodesk, allows users to view the model in full color on all sides. In addition to just viewing the 3D model, users can view a tour or see an abstract about the artifact. One interesting feature allows users to edit the model by changing the color, lighting, and background. These capabilities would be beneficial to educators.

There are several resources other than the models located on this site. The Getting Started section gives users tips and tricks to getting the most out of the site. The Educators section of the site provides historical information, data sets, stories, and a free e-book about scanning and printing the Abraham Lincoln life masks. The Video Gallery provides videos of team members scanning the actual artifacts, discuss- ing the process of turning the scans into 3D models, and other 3D related activities performed at the Smithsonian Institution.

\section{NIH 3D Print Exchange}

The site consists of the following main sections: Discover, Share, Create, Learn, Engage, News \& Events, About, and Search. The interface looks nice and clean. The colors are bright. All the images are high quality. The features and search results on the site are all well organized, easy to identify and use. Users can simply use the top menu, or pick and click on visualized icons to access the subpages. Please refer to the Search section below for the evaluation of the search interface.

To search the content, one can use Discover and Search. These labels would seem to provide similar functions and may cause confusion to the users at the beginning.

Under Discover, users can choose to view Curated Collections, find Builds of 3D models from the users, or search both. 3DPX Collections now consists of special collections sponsored or curated by select collaborators. Now one can search Prosthetics curated by eNABLE, Neuroscience by EyeWire, Heart Library by Jump Simulation, and Molecule of the Month by RCSB Protein Data Bank. 3DPX Builds is a collection of $3 \mathrm{D}$ models from the users, including photos and info on one's own 3D prints.

From the left column on the Discover page, one has a variety of search options. A user can choose to execute a key word search, set more parameters to various categories (such as Medical/Anatomical, Custom Labware, Small Molecules, Proteins/Macromolecules/Virus, and Bacteria/Cell/Tissue/Organism), or choose to search by various

\section{Contact Information \\ NASA \\ NASA Headquarters \\ 300 E. Street SW, Suite 5R30 \\ Washington, DC 20546 \\ Phone: (202) 358-0001 \\ E-mail: <arc-special-proj@lists.nasa.gov> \\ Product URL: <https://nasa3d.arc.nasa.gov> \\ Producer URL: <http://www.nasa.gov/>}

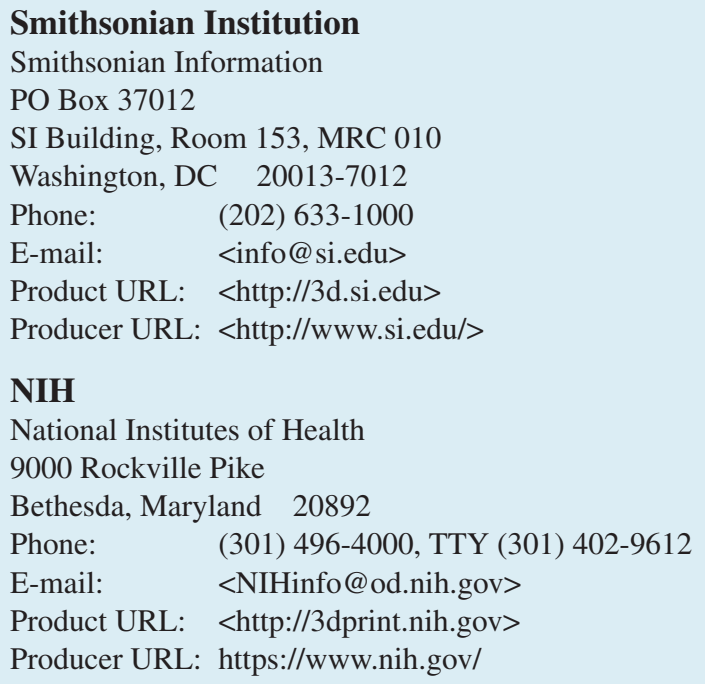


kinds of licenses (such as CC-BY, CC-BY-SA, GNU GPLv3, and so forth). One can change the number of items displayed per page to 24 , 48 , or 72 items per page.

The middle part of the screen on the Discover page shows as default a number of featured 3D printable models arranged in titles, allowing the users to browse and click on any model image or link to explore further. After submitting a search, the middle part of the screen will show the results also arranged in tiles. Then one may choose to display the image in 3D setting, as well as launch a free full screen of interactive 3DPX Model Viewer, where one can manipulate the 3D model on the screen to see different parts from different angles. The software provides technical information of the model, such as the number of FPS, ANIM, TRAVERSE, SORT, RENDER, DRAW, PICKING, NOTES, SHAPES, POINTS, TRIS, and LOADS. QR Code, rating, and licensing information are conveniently available below each image of the model.

Each model also provides detailed metadata information, including what tool was used to generate the model, model ID, category, key words assigned to the model, and the model color. The tool's name in the metadata section is a clickable link to the tool provided by the source for the users to generate similar models. The metadata section includes clickable cross reference link for the users to get related reference information. For example, "Crystal Structure of Foot and Mouth Disease Virus A22 Serotype" was linked to "4GH4" in the Protein Data Bank, which is an information portal to biological macromolecular structures. After viewing a 3D model, the users can choose to print the model out, remix it, or download it. A free NIH 3D Print Exchange account with user name and password are required. One can download the model in WRL, STL, ZIP, and X3D files. The remix feature is a new feature as of January 13, 2015.

Different than Discover, Search allows the users to search broader information on the site. Using Advanced Search on the Search page, one can choose to Search Using Keywords, Search Containing Any of the Words, Containing the Phrase, Search Containing None of the Words, or set limits to search blog, FAQ, forum topic, model, poll, and/or tutorial on the site. Compared to Discover, the design of Search is not very helpful to lay people. There is no or very little help or guidance

Free Text Keywords: Government Information I 3D Printing | Reference | Collection I Technology | Science I Humanities I Social Science I Aeronautics | Bioscience I Museum | Government Agencies

\section{Primary Category: Government Information}

Secondary Categories: Art \& Architecture; History \& Area Studies; Library and Information Science (LIS); Medicine, Nursing \& Health; Multidisciplinary (or interdisciplinary); Philosophy \& Religion; Science, Technology, Computers, Engineering (including Environment); Sociology, Education, Anthropology, Psychology

\section{Type of product being reviewed: Software}

Target Audience: Secondary; General public; Undergraduate (including community colleges); Graduate/Faculty/Researcher

Access: Open Access (OA) next to each search option. Also the search results are pure text and links full of jargon. Images are not shown, making it hard for nonhealth science specialists to understand and make selections. The design of Discover works better for searching. During the process of exploration it always allows searching and browsing functions on the same page. The search results show text and images at the same time. These searching features are really important for searching models.

According to the information found on the site, it is not optimized for Internet Explorer. It works better with Chrome, Firefox, or Safari. The reviewer did not notice much difference using the IE version. The site has an interactive viewer for users to view and manipulate the models. Both WebGL and X3DOM are used for the site's interactive viewers. $\mathrm{X} 3 \mathrm{DOM}$ is an open source framework for viewing $3 \mathrm{D}$ graphics in a web browser (x3dom.org). Most models can be downloaded in STL file format or in Virtual Reality Modeling Language (VRML), a file format that indicates color. There are also other file formats accessible for some models including Blender files, X3D files, and others.

The site welcomes the users to share their own 3D-printable model. Just click on the tab Share on the site. Converting a digital model into a printable format to generate a solid object is not easy. The site claims to provide free novel online tools for generating high-quality and scientifically-relevant 3D-printable models in only minutes, simply by uploading a file or typing in a database accession code. Tutorials on how to generate printable models on the Exchange and submit a data file like medical imaging, molecular data, small molecules, and image stacks are available for researchers and lay people who have little or no experience with using the software to create digital models. Users can click on Create or Learn on the site to access the tutorials.

Under the Engage tab, enthusiastic users can access the Tweets by @NIH3Dprint or visit News \& Events to read information updates. Under About, one can find information about this project and a new section of Scientific Discovery with links to YouTube recordings of interviews with NIH researchers about how they are using 3D printers to support their research. Under About users can also find information on Medical Learning, Additional Resources, Site Policies, and Help $\&$ FAQs. Another new section of STEM Education is under construction.

\section{Critical Evaluation \& Competitive Products}

The government sites offer some unique features and are different than other 3D model repositories available online such as Thingiverse, Repables, and YouMagine. Government repositories are more focused on specific collections and specific subjects. Sites like the Smithsonian Institute only focus on items in the Smithsonian's collection. For the most part, the government websites include curated groups of models. While the NIH does allow users to upload models, the agency indicates clearly labels models which have been NIH verified. Another difference revolves around rights. Most of the nongovernmental repositories encourage users to upload models with a Creative Commons license. The U.S. Governmental websites, for the most part, make the models available in the public domain, free, and void of copyright. (<https://theunitedstates.io/licensing/>)

\section{Purchase \& Contract Provisions}

N/A 


\section{NASA 3D Review Scores Composite: $\star \star \star 1 / 4$}

The maximum number of stars in each category is 5.

\section{Content:}

\section{$\star \star \star 1 / 2$}

The site has a variety of space related models available for 3D printing. The models all originate with NASA and its affiliates ensuring the quality and accuracy. There is not much supplementary material to the models besides some links and articles.

\section{User Interface/Searchability: $\quad \star \star \star \star$}

The site has a simple search box and a straightforward interface. There is a browsable A-Z list of 3D printable items which can be searched by title or date added.
Pricing:
N/A
Purchase/Contract Options:
N/A

\section{Smithsonian X3D Review Scores Composite: $\star \star \star \star 3 / 4$}

The maximum number of stars in each category is 5.

\section{Content:}

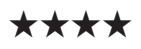

The site contains many models scanned from the Smithsonian's collection along with copious amounts of supplementary material. The site has a strong leaning towards education and provides articles, images, and videos relating to the 3D models and the Smithsonian's artifacts.

\section{User Interface/Searchability: $\quad \star \star \star 1 / 2$}

The site has no search feature. Users can only browse the models. A free account is required to download any of the models. Some of the tabs are confusing and repetitive. It is not intuitive if supplementary resources such as a tour are available for a specific model. The free online viewer to observe the models works very well and has a great design for educators wishing to show and manipulate the models.
Pricing:
N/A

Purchase/Contract Options: $\quad$ N/A

\section{NIH 3D Print Exchange Review Scores Composite: $\star \star \star \star \star$}

The maximum number of stars in each category is 5 .

\section{Content:}

\section{$\star \star \star \star \star$}

The site is for a variety of audiences, from researchers, educators and students, presenters, to 3D printing enthusiasts. The content comes from known curators and the users. This site has growing content and cutting edge 3D technology features, allowing the users to search, browse, download, and share biomedical 3D print files, modeling tutorials and educational material.

\section{User Interface/Searchability}

The design of the interface and the search features of this site are the most comprehensive among the three resources reviewed here.

Among all the three resources reviewed here, the search results on this site also provide the most detailed metadata. The content and features are interactive, well organized, look nice and clean.

Pricing: N/A

Purchase/Contract Options: N/A 


\section{Authentication}

N/A

\section{Authors' References}

Danchilla, B. (2012). Beginning WebGL for HTML5. Berkeley: Apress.

Getting Started. (n.d.). Retrieved from <http://3d.si.edu/article/getting-started>

Holdren, John. Improving the Management of and Access to Scientific Collections. Memorandum for the Heads of Executive Departments and Agencies. Washington, DC: Executive Office of the President, Office of Science and Technology Policy. 2014 March 20, <https:// www.whitehouse.gov/sites/default/files/microsites/ostp/ostp_memo_ scientific_collections_march_2014.pdf>

Legal Nature of the Smithsonian. (n.d.). Retrieved from <https:// www.si.edu/OGC/legalhistory>

Prerequisites. (n.d.). Retrieved from $<$ http://3d.si.edu/article/prerequisites>

Printing up replica artifacts. (2014). Mechanical Engineering, 136(2), 24-25.

Stebbins, M. \& Lieberman, E. (2014). Fossils, Seeds, and Space Rocks: Improving the Management of and Access to the Nation's Sci- entific Collections. Retrieved from <https://www.whitehouse.gov/ blog/2014/03/20/fossils-seeds-and-space-rocks-improving-management-and-access-nation-s-scientific-co $>$

\section{About the Authors}

Jennifer Horton is the Science Librarian at Southern Illinois University at Carbondale, IL. She received her M.L.S. from the University of Missouri-Columbia. She holds a J.D. from Saint Louis University School of Law and a B.S. in Biology from Truman State University. She is the coordinator of the $3 \mathrm{D}$ printing and scanning program at SIUC's Morris Library. Her research interests include 3D printing and other technologies in academic libraries and information literacy in the sciences.

Jian Anna Xiong is the Government Information Librarian/Associate Professor at Southern Illinois University at Carbondale, IL. She received her M.L.S. from the Indiana University Bloomington. She holds a second Master degree in German Language and Literature from Beijing Foreign Studies University, Beijing, P.R. China. She is the manager of selective Federal Depository Library Program and IL State Depository Library Program at SIUC's Morris Library since 2003. She has a continuous record of research publications on government information and other library profession related topics.

\section{Subscribe TO THE CHARLESTON ADVISOR Today!}

\section{The Charleston Advisor: Critical Reviews of Web Products for Information Professionals}

\section{Over 800 Reviews Now Included}

- Libraries pay less than $\$ .40$ per review

- Publishers pay less than $\$ .65$ per review

\section{How Much Are You Spending?}

- Web Database and Quarterly Print are now available at the low price of $\$ \mathbf{2 9 5 . 0 0}$ for libraries; $\mathbf{\$ 4 9 5 . 0 0}$ for all others.

ORDER YOUR SUBSCRIPTION TODAY.

Order on the Web: www.charlestonco.com

\section{YES! Enter my Subscription for One Year.}

Name:

Title:

Organization:

Address:

City/State/Zip:

Phone:Fax:

E-Mail:

$\square$ YES. I am interested in being a Reviewer.

6180 E. Warren Ave., Denver, CO 80222

Phone: (303) 282-9706 Fax: (303)282-9743 